\title{
A View on Stochastic Finite Element and Geostatistics for Resource Parameters Estimation
}

\author{
Skender Osmani ${ }^{1}$, Mihallaq Kotro ${ }^{2}$, Ervin Toromani ${ }^{2}$, Aida Bode $^{1}$, Arben Boçari $^{2}$ \\ ${ }^{1}$ Polytechnic University of Tirana, Tirana, Albania; ${ }^{2}$ Agriculture University of Tirana, Tirana, Albania. \\ Email: s_osmani@yahoo.com, bbocari@yahoo.com
}

Received October $29^{\text {th }}, 2012$; revised November $30^{\text {th }}, 2012$; accepted December $10^{\text {th }}, 2012$

Copyright (C) 2013 Skender Osmani et al. This is an open access article distributed under the Creative Commons Attribution License, which permits unrestricted use, distribution, and reproduction in any medium, provided the original work is properly cited.

\begin{abstract}
The resource parameter estimation using stochastic finite element, geostatistics etc. is a key point on uncertainty, risk analysis, optimization [1-5] etc. In this view, the paper presents some consideration on: 1) Stochastic finite element estimation. The concept of random element is simplified as a stochastic finite element (SFE) taking into account a parallelepiped element with eight nodes in which are given the probability density functions (pdf) on its point supports. In this context it is shown: a - the stochastic finite element is a linear interpolator, related to the distributions given at each nodes; $\mathrm{b}$ - the distribution pdf in whatever point $x \in \mathrm{V}$; $\mathrm{c}$ - the estimation of the mean value of $Z(x)$; 2) Volume integrals calculus; 3) SFE in geostatistics approaches; 4) SFE in PDE solution. Finally, some conclusions are presented underlying the importance of SFE applications
\end{abstract}

Keywords: Parameter Estimation; SFE; Geostatistics; Kriging; Risk Analysis; Optimisation

\section{Introduction}

Many physical phenomena and processes are mathematically modeled by partial differential equations (PDE). The data required by PDE's models as resource and material parameters are in practice subject to uncertainty due to different errors or modeling assumptions, the lack of knowledge and information. In this view the parameters are (not deterministic) stochastic ones [6].

The considerable attention that stochastic finite element (SFE) received over the last decade [7-9] is mainly attributed to the spectacular growth of computing power, rendering possible the efficient treatment of large scale problems in dynamics of processes etc.

Fundamental issue in SFE is the parameter estimation and reserves. The most outstanding method for the approximate solution of a SPDE is the MONTE CARLO method [10]. On the other hand, the geostatistics is a useful discipline to make the inference about the spatial risk phenomenon (processes) [11].

\section{A View on the Random Element}

Let's be defined a fixed probability space $(\Omega, \mathcal{A}, P)$ [7], where $\Omega$ is a nonempty set of "outcomes" or elementary events", $\mathcal{A}$ is a $\sigma$ algebra of subsets of $\Omega$ (the "random events" ) and $P$ is a probability measure on the measur- able space $(\Omega, \mathcal{A})$ If $\left(\chi, S_{x}\right)$ is another measurable space, then a random element $X$ in $\chi$ is a measurable mapping from $(\Omega, \mathcal{A}, P)$ into $\left(\chi, S_{x}\right)$ i.e. it holds $X: \Omega \rightarrow \chi$ with:

$$
X^{-1}(B):=\{X \in B\}:=\{\omega \in \Omega: \chi(\omega) \in B\} \in A, \forall B \in S_{x}
$$

with each random element $\mathrm{X}: \Omega \rightarrow \chi, P_{x}$ is a probability measure of $\left(\chi, S_{x}\right)$ connected with the distribution of random elements. It is defined by:

$$
P_{x}^{\prime}(B):=P\{X \in B\}:=P\{\omega \in \Omega: \chi(\omega) \in B\},\left(B \in S_{x}\right)
$$

A random element $X$ with values in $X$ is called a simple random element if the range is a finite nonempty set in $X$, where exists a partition $[4,12]$ of the probability space $\Omega=\bigcup_{k=1}^{N} \Omega_{k}$ with measurable sets

$$
\Omega_{k} \in A \quad k=1,2, \cdots, N(N \in \mathbf{N})
$$

such like: $X(\omega)=x_{k}$ for $\omega \in \Omega_{k}$.

The corresponding probabilities are:

$$
\begin{gathered}
P\left(\Omega_{k}\right)=p_{k}, p_{k} \geq 0, k=1,2, \cdots, N \\
\sum_{k=1}^{N} p_{k}=1
\end{gathered}
$$

The distribution of a simple random element is a discrete 
probability measure on $\left(X, S_{x}\right)$ that might be written as: $P_{x}=\sum_{k=1}^{N} p_{k} \delta_{x k}$,

where: $\delta_{x k}$ the Dirac measure

$$
\delta_{x k}(B)= \begin{cases}1 & \text { if } x_{k} \in B \\ 0 & \text { otherwise }\end{cases}
$$

\section{Stochastic Finite Element [13]}

Even though this is a general concept $[7,14]$ we will present some considerations in the viewpoint its applications in the parameter estimation of different phenomena and processes.

Let's consider a zone $V \subset R^{3}$ and a random function $Z(x), x \in V$. The zone $V$ is sorted out into blocks $v_{i}$ by a parallelepiped grid:

$$
V=\bigcup v_{i}
$$

where: $v_{i}$ is a parallelepiped element with eight nodes.

At each node, the random function $Z(x)$ is known, in other words is given the probability density function (pdf) on its point support (Figure 1). It is required:

The distribution pdf in whatever point $x \in V$.

The estimation of the mean value

$$
z_{v i}=\frac{1}{v} \int_{V} Z(x) \mathrm{d} x \text { over the domain } v
$$

We define a stochastic element as a block, with the random function $Z(x), x \in v_{i}$.

Let us consider a reference element $w_{i}$ in the co-ordinate system $s_{1} s_{2} s_{3}$. If we choose an incomplete base [15]:

$$
P(s)=\left\langle 1, s_{1}, s_{2}, s_{3}, s_{1} s_{2}, s_{2} s_{3}, s_{3} s_{1}, s_{1} s_{2} s_{3}\right\rangle
$$

Then the function $Z(x)$ could be presented as a linear combination :

$$
Z(x)=Z\left(s_{1} s_{2} s_{3}\right)=\langle P(s)\rangle\left[P_{8}\right]^{-1}\left\{Z_{s}^{8}\right\}=\langle N(s)\rangle\left\{Z_{s}^{8}\right\}
$$

where:

$\left[P_{8}\right]^{-1}$-is the matrix, whose elements are the polynomials base values at the nodes

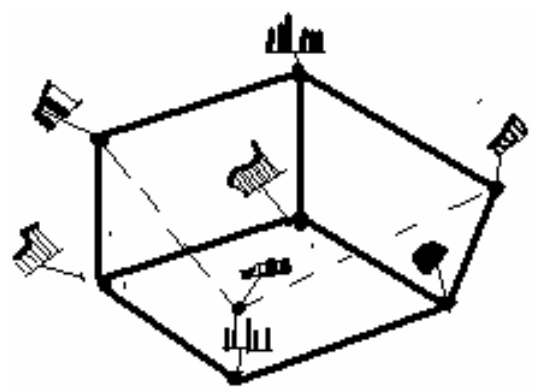

Figure 1. Parallelpiped element.
$\left\{Z_{s}^{8}\right\}$-is the vector of the distributions of the nodes;

$\langle N(s)\rangle$-is the vector of the shape functions;

$$
\begin{gathered}
N_{i}, i=1,2, \cdots, 8 \\
N(s)=\left\langle N_{i}(s)\right\rangle=\left\langle N_{1}(s), N_{2}(s), \cdots, N_{8}(s)\right\rangle \\
N_{i}(s)=\left(1+s_{1} s_{1}^{i}\right)\left(1+s_{2} s_{2}^{i}\right)\left(1+s_{3} s_{3}^{i}\right) \quad i=1,2, \cdots, 8
\end{gathered}
$$

In the formula (5), "the exponent $i$ " is not a variable. It indicates only the sign within the parentheses.

\subsection{The Mean Value}

To calculate the mean value $z_{v i}=1 / v \int_{V} Z(x) \mathrm{d} x$, we consider the deterministic transformation :

$$
X_{i}(s)=\left\langle N_{i}(s)\right\rangle\left\langle x_{i}^{8}\right\rangle \quad i=1,2, \cdots, 8
$$

Therefore [13]

$$
\begin{aligned}
& Z_{v i} \\
& =\frac{1}{v} \int_{v} Z\left(x_{1}\left(S_{1} S_{2} S_{3}\right), x_{2}\left(S_{1} S_{2} S_{3}\right), x_{3}\right. \\
& =\sum_{i=1}^{8} H_{i} Z_{i}(x) \\
& H_{i}=f\left(a_{i j}, b_{i j}, c_{i j}, d_{i j}\right)
\end{aligned}
$$$$
=\frac{1}{v} \int_{v} Z\left(x_{1}\left(S_{1} S_{2} S_{3}\right), x_{2}\left(S_{1} S_{2} S_{3}\right), x_{3}\left(S_{1} S_{2} S_{3}\right)\right)
$$$$
\operatorname{det} J \mathrm{~d} s_{1} \mathrm{~d} s_{2} \mathrm{~d} s_{3}
$$

The coefficients $a_{i j}, b_{i j}, c_{i j}, d_{i j}, i, j=1,2,3$ are depend only on the node coordinates. Knowing the above coefficient we can calculate [13] the weight coefficient $H_{i}(i=1, \cdots, 8)$ as for example for $H_{2}$ :

$$
\begin{array}{rl}
H_{2} & 8 / 3 c_{21} a_{32} c_{13}+8 / 9 d_{21} a_{32} a_{13}+8 / 27 c_{21} c_{32} d_{13} \\
& +8 / 27 d_{21} c_{32} b_{13}+8 / 9 a_{21} b_{32} c_{13}+8 / 9 b_{21} b_{32} a_{13} \\
& +8 / 27 a_{21} d_{32} d_{13}+8 / 27 b_{21} d_{32} b_{13}+8 / 9 c_{11} a_{22} d_{33} \\
& +8 / 9 d_{11} a_{22} a_{33}+8 / 9 a_{11} b_{22} c_{33}+8 / 9 b_{11} b_{22} a_{33} \\
& +8 / 27 c_{11} a_{22} d_{33}+8 / 27 d_{11} c_{22} b_{33}+8 / 27 a_{11} d_{22} d_{33} \\
& +8 / 27 b_{11} d_{22} b_{33}+8 / 9 a_{12} a_{23} d_{31}+8 / 9 b_{21} a_{23} b_{31} \\
& +8 / 27 c_{12} b_{23} d_{31}+8 / 27 d_{12} b_{23} b_{31}+8 / 9 a_{12} c_{32} c_{31} \\
& +8 / 9 b_{12} c_{23} a_{31}+8 / 27 a_{21} d_{32} c_{31}+8 / 27 d_{12} d_{23} a_{31} \\
& +8 / 9 a_{13} a_{22} d_{31}+8 / 9 c_{13} a_{22} c_{31}+8 / 9 a_{13} b_{13} b_{31} \\
& +8 / 9 c_{13} b_{22} a_{31}+8 / 9 b_{13} d_{22} d_{31}+8 / 27 d_{13} c_{22} c_{31} \\
& +8 / 27 b_{13} d_{13} b_{31}+8 / 27 d_{13} d_{22} a_{31}+8 / 9 c_{21} a_{12} c_{33} \\
& +8 / 9 d_{21} a_{12} a_{33}+8 / 9 a_{12} b_{12} c_{33}+8 / 9 b_{21} b_{12} a_{33} \\
& +8 / 27 c_{21} c_{12} d_{33}+8 / 27 a_{13} a_{22} d_{31}+8 / 27 a_{12} d_{12} d_{33} \\
& +8 / 27 b_{21} d_{12} b_{33}+8 / 9 c_{11} a_{32} c_{23}+8 / 9 d_{11} a_{32} a_{23} \\
& +8 / 9 a_{32} b_{32} c_{23}+8 / 27 b_{11} b_{32} a_{13}+8 / 9 c_{11} c_{32} d_{23} \\
& +8 / 27 d_{11} c_{32} b_{23}+8 / 27 a_{32} d_{32} d_{23}+8 / 27 b_{11} d_{32} b_{23}
\end{array}
$$


It is known that:

$$
\sum_{i=1}^{8} H_{i}=1
$$

Thus, the coefficients $\mathrm{H}_{\mathrm{i}}$ are the distribution weights. In other words they make the weighted average of the given distributions at the nodes.

Thus, the mentioned stochastic finite element estimator is a linear interpolator, regarding to the distributions given at its nodes [13].

Taking into account that averaging process is one of the most frequently employed concept in computational techniques at finite element and geostatistics, below are presented two integral estimation procedures, which are key points on the estimation of the stiffness matrices in SFE and kriging, cokriging, covariance matrices in geostatistics $[11,16,17]$.

\section{Volume Integrals within Polyedras [18]}

Let's take a function $\mathrm{u}\left(x_{1}, x_{2}, x_{3}\right)$ in a coordinate system $x_{1}, x_{2}, x_{3}$. The integral of volume $V$ will be estimated:

$$
\int_{v} u\left(x_{1}, x_{2}, x_{3}\right) \mathrm{d} v
$$

We will construct a vector $\hat{\phi}\left(x_{1}, x_{2}, x_{3}\right)$ that will satisfy :

$$
u=\operatorname{div} \hat{\Phi}
$$

where:

$$
\varphi=\varphi_{1} \hat{i}_{1}+\varphi_{2} \hat{i}_{2}+\varphi_{3} \hat{i}_{3}
$$

$i_{1}, i_{2}, i_{3}$ is the system of the unit vector along the coordinate directions.

Let's suppose that the boundary surface $S$ of the volume $V$ is composed of k plane polygonal faces $S_{i}(i=1$, $2, \cdots, k)$. Applying the divergence theorem we find:

$$
\int_{v} u\left(x_{1}, x_{2}, x_{3}\right) \mathrm{d} v=\sum_{j=1}^{k} \iint u\left(x_{1}, x_{2}, x_{3}\right) \mathrm{d} x_{1} \mathrm{~d} S_{j}^{1}
$$

where: the projected area $\mathrm{d} S_{j}^{1}$ is perpendicular to $\hat{i}$ and lies in the $\left(x_{2}, x_{3}\right)$ plane.

The equation of the plane face $\mathrm{d} S_{j}$ can be expressed as:

$$
x_{1}=x_{1}^{(j)}\left(x_{2}, x_{3}\right)=\alpha_{1}^{(j)}+\alpha_{2}^{(j)} x_{2}+\alpha_{3}^{(j)} x_{3}
$$

so the right-hand side of Equation (12) can be simplified to be :

$$
\int_{V} u\left(x_{1}, x_{2}, x_{3}\right) \mathrm{d} V=\sum_{j=1}^{k} \int_{S_{j}} \varphi^{(j)}\left(x_{1}, x_{2}\right) \mathrm{d} S_{j}
$$

where: the surface $S_{j}^{1}$ is a polygon in the $\left(x_{2}, x_{3}\right)$, in which the function $\varphi^{(j)}$ is to be integrated for $\bar{j}=$ $1,2, \cdots, k$.

In this way, the computation of the volume integral is a procedure to integrate an arbitrary function within a polygon. Further repeating the above mentioned procedure we could find:

$$
\begin{aligned}
\int_{\Omega} V\left(x_{1}, x_{2}\right) \mathrm{d} \Omega & =\int_{T} \Psi\left(x_{1}, x_{2}\right) n_{1} \mathrm{~d} T=\sum_{j=1}^{k} \int_{T} \Psi\left(x_{1}, x_{2}\right) n_{1} \mathrm{~d} T \\
& =\sum_{j=1}^{k} \int_{\Gamma_{j}} \Psi\left(x_{1}^{(1)}, x_{2}\right) n_{j}^{(j)} \mathrm{d} T
\end{aligned}
$$

where, the perimeter $T$ is a collection of the straight lines

$$
T_{j}, j=1, \cdots, k,
$$

while,

$$
\begin{gathered}
x_{1}=x_{j}^{(j)}\left(x_{2}\right) \\
n_{1}^{(j)} \mathrm{d} T=\mathrm{d} x_{2}
\end{gathered}
$$

Let the $x_{2}$ coordinates for $j^{\text {th }}$ side $x_{2}^{j s}$ and $x_{2}^{j e}$. So:

$$
\begin{aligned}
\int_{T_{j}} \Psi_{1}\left(x_{1}, x_{2}\right) n_{1} \mathrm{~d} T & =\int_{x_{2}^{j s}}^{x_{2}^{j e}} \Psi\left(x_{1}^{(j)}\left(x_{2}\right), x_{2}\right) \mathrm{d} x_{2} \\
& =\int_{x_{2}^{j s}}^{x_{2}^{j e}} \gamma_{j}\left(x_{2}\right) \mathrm{d} x_{2}
\end{aligned}
$$

Finally the above integral could be estimated by the Gaussian scheme quadrature. It is to be noted that volume integral is a deterministic procedure, but if the $\omega=$ $v$ and $X(\omega)=u$, then it could be estimated as a stochastic finite element using Monte-Carlo method.

Parallelly if $u(x), x \in R^{n}$ is a random function (RF) then the integral $1 / v \int_{V} u(x) \mathrm{d} v$ could be treated in the geo-statistical view as a mean value.

\section{Geostatistical Approach}

\subsection{Variograms}

Geostatistics are based on the theory of the regionalized variables [2] with assumption that data are observations of stochastic variables. The central tool of geostatistics is the variogram or semivariance function which is a structure describing the spatial dependence of the spatial variable [11].

The following formula is the most frequently used for the variogram (semivariance) calculations:

$$
\gamma(h)=\frac{1}{2 N} \sum_{i=1}^{N}\left[Z\left(x_{i}\right)-Z\left(x_{i}+h\right)\right]^{2}
$$

where:

$x_{i}$ is a data location, $h$ is a $\log$ vector, $z\left(x_{i}\right)$ is the data value at location $x_{i}, N$ is the number of data pairs spaced a distance and direction $h$ units apart

Semivariance calculations can also be performed with 
data from RS images for example as a cross variogram. It is defined as half of the average product of the log distance relative to the two variables $Z$ and $Y$.

$$
\begin{aligned}
& \gamma_{z y}(h) \\
& =\frac{1}{2 N} \sum_{i=1}^{n(h)}\left\{\left[Z\left(x_{i}\right)-Z\left(x_{i}+h\right)\right]\left[Y\left(x_{i}\right)-Y\left(x_{i}+h\right)\right]\right\}
\end{aligned}
$$

where:

$Z\left(x_{1}\right)$ and $Y\left(x_{1}\right)$ are the data value in point $x_{1}$ for two bands (profiles);

$N$ is the number of data separated by length of the vector $h$;

$A$ variogram usually is characterized by three parameters [2]:

- Sill- the platean that the semivariogram reaches;

- Range - the distance at which two data points are uncorrelated;

- Nugget - the vertical discontinuity at the origin.

Usually the application of the semivariograms requires that the data accomplish the intrinsic hypothesis for a regionalized variable. In other words a random function $Z(x)$ is said to be intrinsic when:

the mathematical expectation exists and does not depend on the support $x$

$$
E\{Z(x)\}=m \forall(x)
$$

for all vectors $h$ the incerement $Z(x+h)-Z(x)$ has a finite variance which does not depend on $x$

$$
\operatorname{Var}[Z(x+h)-Z(x)]=E\left\{[Z(x+h)-Z(x)]^{2}\right\} \forall(x)
$$

where:

$Z(x)$ is a random function i.e. locally at a point $x_{1}, Z\left(x_{1}\right)$ is a random variable and $Z\left(x_{1}\right)$ and $Z\left(x_{1}+h\right)$ are generally independent but are related by a correlation expressing the spatial structure of the initial regionalized variable $Z(x)$. Experimental variogrames are approximated by different models like: spherical, exponential, Gaussian, circular, tetraspherical, pentaspherical, Hole effect, $K-$ Bessel etc. $[2,16,18]$.

\subsection{Kriging in SFE View [13]}

Let be $Z(x)$ the random function and the estimation of the mean value:

$$
Z_{V}=\frac{1}{v} \int_{V} Z(x) \mathrm{d} x
$$

over a given domain $v$ is required knowing a support of discrete values $Z_{\alpha}, \alpha=1, \cdots, n$.

According to the Kriging approach [2] the linear estimator $Z_{k}^{*}$ of the $\mathrm{n}$ data values is considered:

$$
Z_{k}^{*}=\sum_{\alpha=1}^{n} \lambda_{\alpha} Z_{\alpha} \quad Z_{\alpha}=\frac{1}{v_{\alpha}} \int_{v_{\alpha}} Z(x) \mathrm{d} x
$$

The $n$ weights $\lambda_{\alpha}$ are calculated under the classic hypothesis of the moments:

$$
\begin{gathered}
E\{Z(x)\}=m \\
E\{Z(x+h) Z(x)\}-m^{2}=C(h) \text { or } \\
E\{Z(x+h)-Z(x)\}^{2}=2 \gamma(h)
\end{gathered}
$$

We must be assure that the estimator is unbiased as well as the variance is minimal. Let us suppose that one (or both) of two hypotheses are not accomplished and both the expectation of $Z(x)$ and the covariance depend on $x$ :

$$
\begin{gathered}
E\{Z(x)\}=m(x) \\
C(x, h)=E\{Z(x+h) Z(x)\}-m(x+h) m(x)
\end{gathered}
$$

Before taking into consideration this hypothesis, it should be underlined, whatever the moment functions are going to be, they should always lead to a positive variance. Also, we will show the calculation of Kriging solution using SFE but without considering its existence and uniqueness (It is not the aim of this paper). To ensure that estimator is unbiased we impose the condition:

$$
\sum_{\alpha=1}^{n} \lambda_{\alpha} m_{\alpha}-m_{v}=0
$$

With

$$
\begin{gathered}
m_{v}=E\{Z v(x)\}=E\left\{\frac{1}{v} \int_{V} Z(x) \mathrm{d} x\right\} \\
m_{\alpha}=E\left\{Z\left(v_{\alpha}\right)\right\}=E\left\{\frac{1}{v_{\alpha}} \int_{V_{\alpha}} Z(x) \mathrm{d} x\right\} \quad \alpha=1, \cdots, n
\end{gathered}
$$

The estimation variance is:

$$
E\left\{\left[Z_{v}-Z_{k}^{*}\right]^{2}\right\}=E\left\{Z_{v}^{2}\right\}-2 E\left\{Z_{v} Z_{k}^{*}\right\}+E\left\{Z_{k}^{*}\right\}
$$

Taking into account the expression of $E\left\{Z_{v}^{2}\right\}$ we have:

$$
\begin{aligned}
E\left\{Z_{v}^{2}\right\} & =E\left\{\frac{1}{v^{2}} \int \mathrm{d} x \int Z(x) Z(y) \mathrm{d} y\right\} \\
& =\sum_{i=1}^{8} \sum_{i=1}^{8} c_{i, j} E\left\{Z_{v}\left(x_{i}\right) Z_{y}\left(y_{i}\right)\right\}
\end{aligned}
$$

Also

$$
\begin{aligned}
& \sum_{i=1}^{8} \sum_{i=1}^{8} c_{i, j} \cdot E\left\{Z_{v}\left(x_{i}\right) Z_{y}\left(y_{i}\right)\right\} \\
& =\sum_{i=1}^{8} \sum_{i=1}^{8}\left\{c_{i, j}\left[C\left(v_{i}, v_{j}\right)+m_{v i} m_{v j}\right]\right\} \\
& =\stackrel{v}{C}(v, v)
\end{aligned}
$$


$m_{v i}$ is the expectation of $Z\left(x_{i}\right)$ at the node $\boldsymbol{i}$,

$\stackrel{v}{C}(v, v)$ is the covariation depending not only by the distance $\boldsymbol{h}$, but also on $\boldsymbol{x}$.

Carrying out other means and substituting to the estimated variance we obtain:

$$
\begin{aligned}
E\left\{\left[Z_{v}-Z_{k}^{*}\right]^{2}\right\}= & \stackrel{v}{C}(v, v)-2 \sum_{\alpha=1}^{n} \lambda_{\alpha} \stackrel{v}{C}\left(v, v_{\alpha}\right) \\
& +\sum_{\alpha=1}^{n} \lambda_{\alpha} \sum_{\beta=1}^{n} \lambda_{\beta} \stackrel{v}{C}\left(v_{\beta}, v_{\alpha}\right)
\end{aligned}
$$

Now the problem is to find the weights $\lambda_{\alpha}$, $\alpha=1, \cdots, k$ which minimize the estimation under nonbias conditions:

$$
\sum_{\alpha=1}^{n}\left(\lambda_{\alpha}-\frac{1}{n}\right) m_{\alpha}=0
$$

For this reason, we use the Lagrange multiplier's method, according to which we need to take the derivatives of:

$$
\begin{aligned}
F= & \stackrel{v}{C}(v, v)-2 \sum_{\alpha=1}^{n} \lambda_{\alpha} \stackrel{v}{C}\left(v, v_{\alpha}\right) \\
& +\sum_{\alpha=1}^{n} \lambda_{\alpha} \sum_{\beta=1}^{n} \lambda_{\beta}\left(v_{\beta}, v_{\alpha}\right)+2 \mu \sum_{\alpha=1}^{n}\left(\lambda_{\alpha}-\frac{1}{n}\right) m_{\alpha}
\end{aligned}
$$

This procedure provides the Kriging system of $n+1$ linear equation equations in $\left(\lambda_{\alpha}, \mu\right)$ :

$$
\begin{aligned}
\sum_{\alpha=1}^{n} \lambda_{\alpha} \stackrel{v}{C}\left(v_{\beta}, v_{\alpha}\right)-\mu m_{\alpha} & =\stackrel{v}{C}\left(v, v_{\alpha}\right) \\
\sum_{\alpha=1}^{n} \lambda_{\alpha} m_{\alpha}=e, \quad e & =\sum_{\alpha=1}^{n} \frac{1}{n} m_{\alpha}
\end{aligned}
$$

which can be expressed in matrix form:

$$
\begin{gathered}
{[K]\left\{\lambda_{\alpha}\right\}=\{M\}} \\
{[K]=\left[\begin{array}{cccc}
c\left(v_{1} v_{1}\right) & c\left(v_{1} v_{2}\right) & \cdots & c\left(v_{1} v_{n}\right) \\
c\left(v_{2} v_{1}\right) & c\left(v_{2} v_{2}\right) & \cdots & c\left(v_{2} v_{n}\right) \\
\vdots & \vdots & \ddots & \vdots \\
c\left(v_{n} v_{1}\right) & c\left(v_{n} v_{2}\right) & \cdots & c\left(v_{n} v_{n}\right)
\end{array}\right]} \\
\{\lambda\}=\left\{\begin{array}{c}
\lambda_{1} \\
\lambda_{2} \\
\vdots \\
\lambda_{n}
\end{array}\right\}\{M\}=\left\{\begin{array}{c}
c\left(v_{1} v\right) \\
c\left(v_{2} v\right) \\
\vdots \\
c\left(v_{n} v\right)
\end{array}\right\}
\end{gathered}
$$

Let us suppose that solution of system (33) exists and it is unique. In this situation, it is quite clear that system (33) is general, in the sense of so-called Kriging system.

\section{Example 1}

In Figure 2 it is shown a structure with 3 blocs: $v_{1}=1$ $\times 1, v_{x}=1 \times 1, v_{2}=2 \times 2$ in a contaminated (radioactive, oil, gas etc.) zone.
The equation of the variogram is $\gamma(x)=4 h$ and the means of the parameter measured in the blocs $v_{1}, v_{2}$ are respectively:

$$
E\left(Z_{1}(x)\right)=0.590 \quad E\left(Z_{2}(x)\right)=0.409 .
$$

Let's estimate the parameter $Z(x)$ in the block $v_{x}$ resolving the Kriging system using finite element.

According to Kriging approach we have: $Z=\lambda_{1} Z_{1}+\lambda_{2} Z_{2}$ where $\lambda_{1}, \lambda_{2}$ parameters of the Kriging system:

$$
\left[\begin{array}{ccc}
\bar{\gamma}\left(v_{1}, v_{1}\right) & \bar{\gamma}\left(v_{1}, v_{2}\right) & 1 \\
\gamma\left(v_{2}, v_{1}\right) & \gamma\left(v_{2}, v_{2}\right) & 1 \\
1 & 1 & 0
\end{array}\right]\left[\begin{array}{c}
\lambda_{1} \\
\lambda_{2} \\
\lambda_{3}
\end{array}\right]=\left[\begin{array}{c}
\gamma\left(x_{1} x_{x}\right) \\
\gamma\left(x_{2} x_{x}\right) \\
1
\end{array}\right]
$$

The solution is $\lambda_{1}=0.5906, \quad \lambda_{2}=0.409, \quad \lambda_{3} \approx 0$.

Therefore,

$$
Z=Z=\lambda_{1} Z_{1}+\lambda_{2} Z_{2}=0.5906 \times 5+0.409 \times 7=5.81 .
$$

\section{Example 2}

In the Figure 3, it is presented a profile in a waste zone in which a parameter has been measured using a constant step $h$.

The respective variogram shown in Figure $\mathbf{4}$ has been approximated by a spheric model:

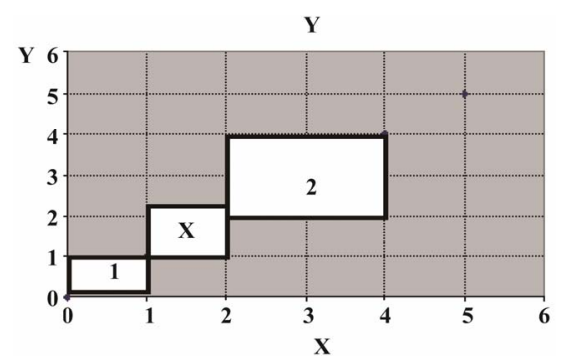

Figure 2. Contaminated zone with three blocks.

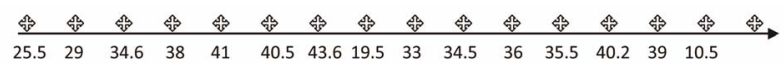

Figure 3. Profile of measured parameter.

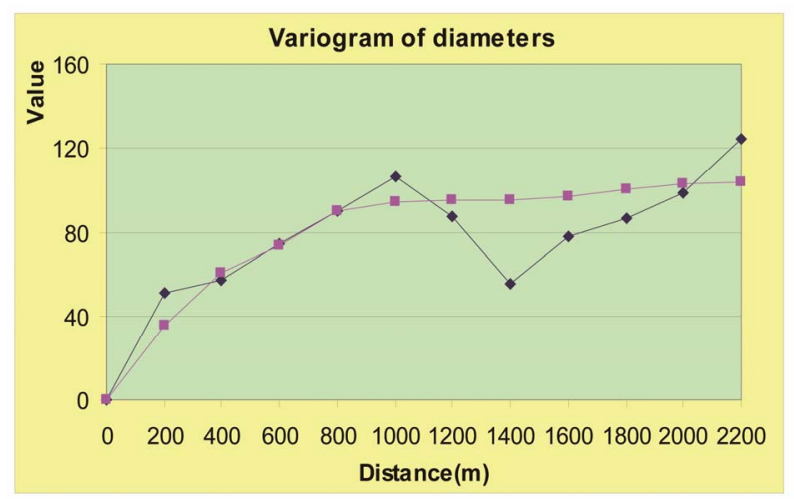

Figure 4. Variogram of diameters depending on distance between sample plots. 


$$
\gamma(h)= \begin{cases}c\left(\frac{3 h}{2 a}-\frac{1}{2} \frac{h^{3}}{a^{3}}\right) & h \leq a \\ 0 & h \geq a\end{cases}
$$

with $c=1$ and $a=4 h$ (the range).

The parabolic form of the variogram around the origin shows it is homogeneous [2].

\section{SFE in Partial Differential Equations}

The parameters of partial differential equations in many cases are not deterministic but stochastic ones. In this view let's have a look on a PDE. First our starting point is the second order elliptic boundary value problem:

$$
\begin{array}{ll}
-\bar{V} *(T \bar{V} p)=F & \text { in } D \\
p=g & \text { on } \partial D_{D} \neq 0 \\
n *(T \overline{V p})=0 & \text { on } \partial D_{N}
\end{array}
$$

posed on a bounded polygonal domain $D \subset R^{2}$, whose boundary is divided into two parts, $\partial D=\partial D_{D} \cup \partial D_{N}$ (Dirichle and Neumman). This steady state diffusion problem can be reformatted by introducing the variable $u=-T \bar{V} p$ as:

$$
\begin{array}{ll}
T^{-1} u+\bar{V} p=0 \\
\bar{V} * u=F & \text { in } D \\
p=g & \text { on } \partial D_{D} \\
n * u=0 & \text { on } \partial D_{D}
\end{array}
$$

In the context of groundwater flow modeling the variable $p$ is the hydraulic head and $\boldsymbol{u}$ is the volumetric flux, respectively.

In many applications, only limited information about the diffusion coefficient $\mathrm{T}$ or the source term $F$ is available.

We assume $T=t(x, \omega)$ (and $F=F(x, \omega)$ to be random fields, i.e. a family of random variables $T(x, \omega)$ with index variable $x \in \bar{D}$. Each random variable takes on values in $\mathbb{R}$ and is defined on a complete probability space $(\Omega, \mathfrak{A}, P)$, where $\Omega$ denotes the set of elementary events, $\mathfrak{A}$ is a $\sigma$-algebra on $\Omega$ generated by the random variables $T(x$,$) , (and F(x)$,$) and P$ is a probability measure.

A consequence of the randomness in the diffusion coefficient or source term is that the output variables $p$ and, if present, $\boldsymbol{u}$ are random fields as well. The primal formulation [12] transforms to the problem of finding a random field:

$$
\begin{array}{cc}
u=u(x, \omega), \quad p=p(x, \omega), \text { such that, } P \text { almost surely } \\
\bar{V}^{*}(T(x, \omega) \bar{V} p(x, \omega))=F(x, \omega) & \text { in } D \times \Omega \\
p(x, \omega)=g(x) & \text { on } \partial D_{D} \times \Omega \quad(36) \\
n *(T(x, \omega) \bar{V} p(x, \omega))=0 & \text { on } \partial D_{N} \times \Omega
\end{array}
$$

Analogously in the mixed formulation [12] we now look for random fields $u=u(x, \omega)$ and $p=p(x, \omega)$ such that: $p$-almost surely (as):

$$
\begin{array}{ll}
T_{-1}(x, \omega) u(x, \omega)+\bar{V} p(x, \omega)=0 \\
\bar{V} u(x, \omega)=F(x, \omega) & \text { in } D \times \Omega \\
p(x, \omega)=g(x) & \text { on } \partial D_{D} \times \Omega \\
n * u(x, \omega)=0 & \text { on } \partial D_{N} \times \Omega
\end{array}
$$

As a simple example let's take a glance at the stochastic finite element on diffusion-convection equation $[5,6,12,19]$ :

$$
\begin{aligned}
& \frac{\partial}{\partial x}\left(k_{x} \frac{\partial \varphi}{\partial x}\right)+\frac{\partial}{\partial y}\left(k_{y} \frac{\partial \varphi}{\partial y}\right) \\
& +V_{x} \frac{\partial \varphi}{\partial x}+V_{y} \frac{\partial \varphi}{\partial y}+Q=C \frac{\partial \varphi}{\partial t}
\end{aligned}
$$

using the Crack-Nickolson algorithm with $0<\theta \leq 1$ :

$$
\begin{aligned}
& \varphi_{i, j}^{n+1} a_{1} \varphi_{i-1, j}^{n+1}+a_{2} \varphi_{i, j}^{n+1}+a_{3} \varphi_{i+1, j}^{n+1} \\
& +a_{4} \varphi_{i, j-1}^{n+1}+a_{5} \varphi_{i, j+1}^{n+1}=\varphi_{i, j}^{n}+b Q_{i, j}^{n}
\end{aligned}
$$

where :

$C$-the solute concentration, $x, y$-spatial co-ordinate, $t$-time coordinate, $V$-the flow velocity vector with its components $V_{x}, V_{y}, D$ - the diffusion coefficient, $a_{i}, i=\overline{1,5}$ and $\mathrm{b}$ are the coefficients depending on the mentioned coefficients, $\Delta x, \Delta y$ spatial steps, $\Delta t$ time step. Below we are presenting a river plane zone contaminated by a point pollutant source Figure 5, placed in the left side of the node 13 .

In this scheme, it was operated with mean values of the random diffusion convection parameters, resulting from their synthetic and real distributions.

The components $V_{x}$ and $V_{y}$ has been measured in an interval of time. The component of $V_{y}$ is positive over the line $13-18$ and negative under this one.

To illustrate the idea, it is shown below a partial solution of the contaminant concentration in the step $s t=5$ for a simple non stationary flow problem (DirichleNewman conditions). Using $q=1, K_{x}=1, K_{y}=1, V_{x}=1$,

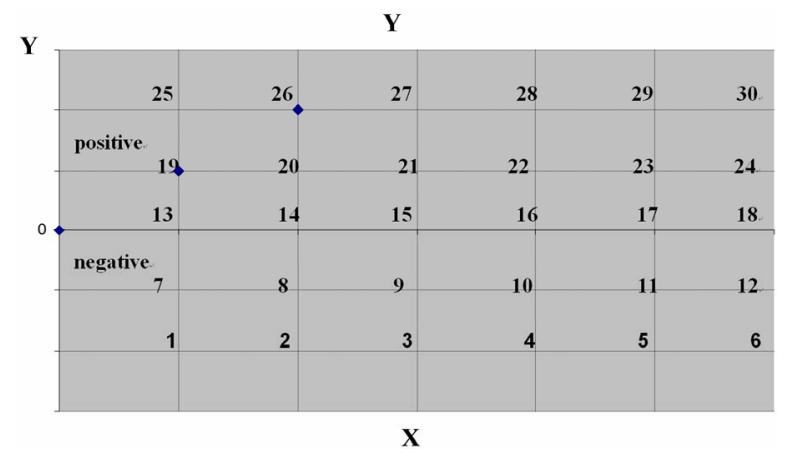

Figure 5. A contaminated river zone. 

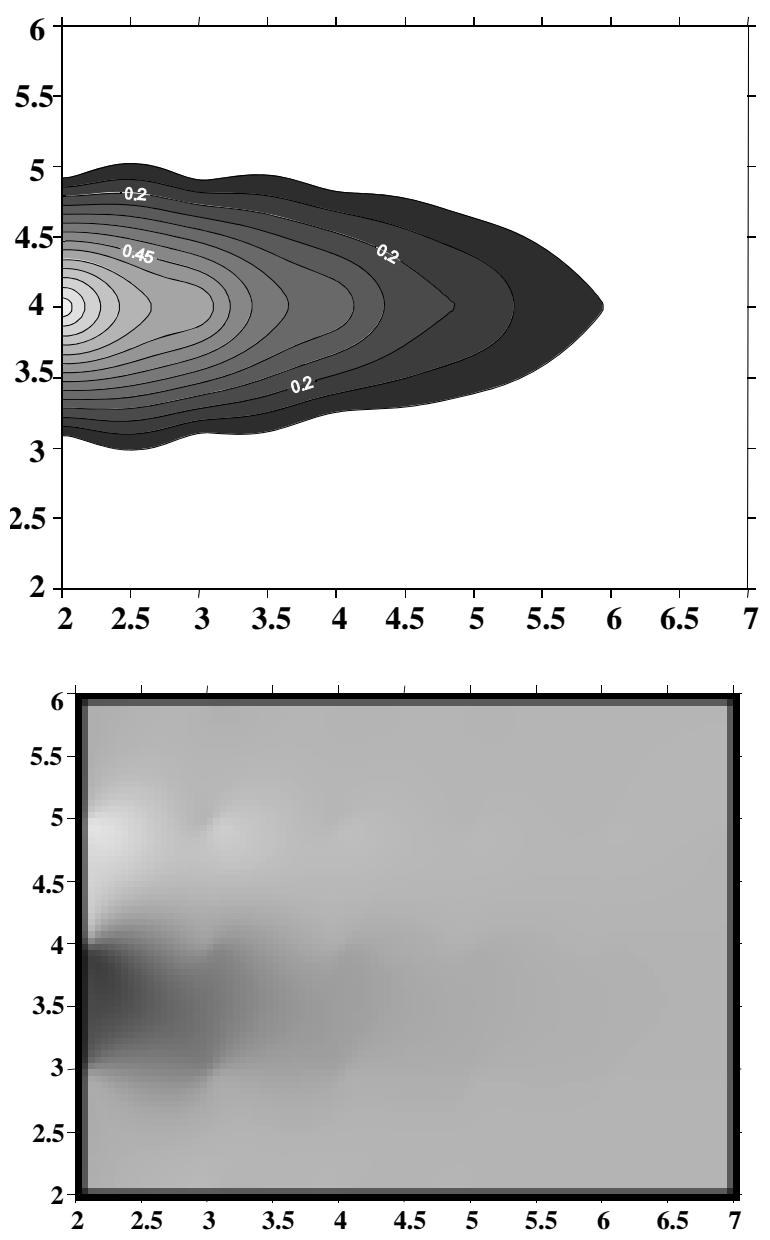

Figure 6. The contaminant concentration dynamic.

$V_{y}=0.01$, the following dimensionless means values by a Monte Carlo procedure [10] resulted:
$\mathrm{xx}[1]=0.0012$
$\mathrm{xx}[2]=0.003$
$\mathrm{xx}[3]=0.004$
$\mathrm{xx}[4]=0.0043$
$\mathrm{xx}[5]=0.0036$
$\mathrm{xx}[6]=0.0029$
$\mathrm{xx}[7]=0.0410$
$\mathrm{xx}[8]=0.069$
$\mathrm{xx}[9]=0.074$
$\mathrm{xx}[10]=0.073$
$\mathrm{xx}[11]=0.051$
$\mathrm{xx}[12]=0.053$
$\mathrm{xx}[13]=0.9000 \quad \mathrm{xx}[14]=0.770$
$\mathrm{Xx}[15]=0.590$
$\mathrm{xx}[16]=0.450 \quad \mathrm{xx}[17]=0.250$
$\mathrm{xx}[18]=0.290$
$\mathrm{xx}[19]=0.0410 \quad \mathrm{xx}[20]=0.069$
$\mathrm{xx}[21]=0.074$
$\mathrm{xx}[22]=0.073 \quad \mathrm{xx}[23]=0.051$
$\mathrm{xx}[24]=0.053$
$\mathrm{xx}[25]=0.0012 \quad \mathrm{xx}[26]=0.003 \quad \mathrm{xx}[27]=0.0041$
$\mathrm{xx}[28]=0.0043 \quad \mathrm{xx}[29]=0.0036 \quad \mathrm{xx}[30]=0.0029$

In Figure 6, it is presented the contaminant concentration dynamic for different times of the flow.

As it was expected the solution is symmetric.

There are simple resemblances between different concepts and operators in geostatistics and SFE as for example: blocs, interpolation operator, minimization of the variance (energy).

\section{Conclusion}

SFE and Geostatistic applications are of the great impor- tance in environmental resources, nuclear and renewable energy, ecology, forestry, geology, climate, water and air pollution, mapping as well as on their uncertainty, risk analysis and optimization [1,5,14,15,20,21].

\section{REFERENCES}

[1] C. Busby, et al., "2010 ECRR Recommendations of the European Committee on Radiation Risk Brussels," BELGIUM, 2010.

[2] A. G. Journel and Ch. J. Huijbreght, "Mining Geostatistics," Academic Press, London, 1979.

[3] E. Guastaldy, "Geostatistical Modeling of Uncertainty for the Risk Analysis of a Contaminated Site in the Northern Italy," Geophysical Research Abstract, European Geoscience Union, Vol. 7, 2005.

[4] M. Kleiber and T. D. Hien, "The Stochastic Finite Element Method," Wiley, Chichester, 1992.

[5] S. Osmani, "Some Consideration on Finite Element Solution of Diffusion-Convection Equation and Its Application on Water Pollution," Proceedings of Internaional Conference on Water Problem in the Mediterranean Countries, North Cyprus, 1997.

[6] S. Osmani and M. Qirko, "Stochastic Finite Elements in a Complex System: Nonstationary Fluid Flow, Mass Transport, Heat Conduction and Vibrating," International Conference in Scaling up in Fluid Flow in Porous Medium CROATIA 2008 as well as (Second Part) in 5th Asian Rock, Symposium Tehran, Tehran, 2008.

[7] R. Ghanem and P. Spanos, "Stochastic Finite Elements: A Spectral Approach," Springer-Verlag, Berlin, 1991.

[8] R. Ghanem, "Ingredients for a General Purpose Stochastic Finite Element Formulation," Computer Methods in Applied Mechanics and Engineering, Vol. 168, No. 19, 1999.

[9] A. Keese, "A Review of Recent Developments in the Numerical Solution of Stochastic Partial Differential Equations (Stochastic Finite Elements)," Institut für Wissenschaftliches Rechnen, Brunswick, 2003.

[10] K. Saberfield, "Monte Carlo Methods for Stochastic PDEs," Institute for Applied Analysis and Stochastic, Berlin, 2007.

[11] J. P. Chiles and P. Delfiner, "Geostatistics, Modeling Spatial Uncertainty," Wiley, New York, 1999. doi:10.1002/9780470316993

[12] A. Bode and S. Osmani, "Using Stochastic Finite Element in Geostatistics and Difusion Convection Equations.

MAMERNO9," Third International Conference on Approximation Methods and Numerical Modeling in Environment and Natural Resources, Pau, 8-11 June 2009.

[13] S. Osmani, "Energy Distribution Estimation Using Stochastic Finite Element," Renewable Energy, Elsevier, London, 2002.

[14] G. Stefanou, "The Stochastic Finite Element Method: Past, Present and Future," Computer Methods in Applied Mechanics and Engineering, Elsevier, London, 2009.

[15] O. C. Zienkiewitz and R. L. Taylor, "The Finite Element 
Method," 5th Edition, The Basic, Elsevier, Oxford, 2000.

[16] P. Govartes, "Geostatistics for Natural Resources Evaluation," Oxford University, New York, 1997.

[17] S. Osmani, "Finite Element on Geostatistique Calculations," In: A. G. Fabri et Royer, Ed., 3rd International Codata Conference in Geomathematics and Geostatistics Science de la Terre, Nancy, 1994.

[18] G. Dasgupta, "Integration within Polygonal Finite Elements," Journal of Aerospace Engineering, Vol. 16, No. 1, 2003, pp. 9-18.
doi:10.1061/(ASCE)0893-1321(2003)16:1(9)

[19] S. Osmani, "Finite Element Method (in Albanian)," Tirana, 2009.

[20] Nuclear Energy, "The Future Climat,” The Royal Society, Portsmuth, 1999.

[21] S. Osmani, "A Model of Convection Diffusion Equation in 3D with Finite Elements," 17th International Biennal Conference Numerical Analysis, Dundee, 1997. 